\title{
Understanding Researchers' Use of a Large, High-Resolution Display Across Disciplines
}

\author{
Fateme Rajabiyazdi ${ }^{1}$, Jagoda Walny ${ }^{1}$, Carrie Mah ${ }^{1}$, John Brosz ${ }^{2}$, Sheelagh Carpendale ${ }^{1}$ \\ ${ }^{1}$ Department of Computer Science, ${ }^{2}$ Libraries and Cultural Resources \\ University of Calgary \\ \{frajabiy, jkwalny, cmah, jdlbrosz, sheelagh\}@ucalgary.ca
}

\begin{abstract}
A driving force behind the design of increasingly large and high resolution displays (LHRDs) has been the need to support the explosion of data in the natural sciences such as physics, chemistry, and biology. However, our experience with an LHRD accessible to researchers across multiple disciplines has shown that they are useful for a wide range of research activities involving large images and data. We conducted in-context, semi-structured interviews with researchers from a variety of disciplines about their experiences using the LHRD with their own data. Notably, it became apparent that the size and resolution of the LHRD supported a multitude of activities related to observation, for which zooming or other enlargement methods on standard resolution screens were not sufficient. The interview findings lead to implications for further research into supporting a broader range of disciplines in using large, high-resolution displays.
\end{abstract}

\section{Author Keywords}

large, high-resolution displays; multi-disciplinary; research support; observation; education; qualitative study.

\section{ACM Classification Keywords}

H.5.m. Information Interfaces and Presentation: Miscellaneous

\section{INTRODUCTION}

Over the last few years, large, high-resolution displays (LHRD) have become less costly and easier to construct [17] and therefore accessible to a wider population. The resulting displays with pixel counts in the tens of millions allow for more, larger, and higher resolution digital objects, such as images, visualizations, and other types of files, to be viewed at a larger scale and in more detail than on standard displays. LHRDs have been shown to have various benefits, such as supporting team work and analysis of large or complex data $[2,10,19,20]$, where increased performance and satisfaction has been compared to working on typical desktop

Permission to make digital or hard copies of all or part of this work for personal or classroom use is granted without fee provided that copies are not made or distributed for profit or commercial advantage and that copies bear this notice and the full citation on the first page. Copyrights for components of this work owned by others than ACM must be honored. Abstracting with credit is permitted. To copy otherwise, or republish, to post on servers or to redistribute to lists, requires prior specific permission and/or a fee. Request permissions from permissions@acm.org. ITS 2015, November 15-18, 2015, Funchal/Madeira, Portugal. Copyright is held by the owner/author(s). Publication rights licensed to ACM. ACM 978-1-4503-3899-8/15/11\$15.00.

Include the DOI: http://dx.doi.org/10.1145/2817721.2817735 displays. Most of these benefits have been investigated from the perspective of natural scientists and data analysts working with large, complex datasets [7], and many, though not all, of these studies have relied on examining performance or satisfaction with pre-defined tasks [2, 10, 19, 24, 29].

However, once a new technology becomes available for more widespread use, it often gets used in ways that were not initially expected or intended. To gain a broader understanding of how LHRDs are actually used, we investigate the naturally arising usage of an LHRD "in the wild" that is available to researchers on a university campus. We conducted a qualitative study using in-context semi-structured interviews of researchers working in a variety of disciplines. We found that researchers from many fields of study other than the natural sciences are making considerable use of this display, in particular for observation-based research. Moreover, they are making important, publishable discoveries on the LHRD that they were not able to make with the use of standard resolution displays or projectors.

Our participants' perspective on how the LHRD can support their own research and related activities centered around the themes of immersive observation, observation strategies, collaboration, and education. They also shared with us discoveries they made on the LHRD: verified, publishable discoveries; some fledgling discoveries; and opportunities for design improvements. We discuss how observation was key to the discoveries made, how the size and resolution of the display affected the tasks performed, and the ways in which participants wanted to use the display. These findings lead to implications for further research into supporting this broader range of disciplines in using large, high-resolution displays.

\section{RELATED WORK}

There has been a consistent desire to use displays that are bigger and have ever higher pixel counts. This has taken various forms ranging from the development of IMAX and OMNIVAX [22] theatres, to the development of CAVEs [11], and to large, high resolution display walls made up of tiled displays [1]. Within the past two years, NVIDIA and ATI have released commercial graphics hardware capable of creating a composite display that allows 16 individual displays to behave as a single display. This makes it possible to run an LHRD from a single machine, rather than as previously when it was necessary to resort to using clusters of comput- 
ers. Using clusters of computers needs parallelization that makes running "normal" applications challenging.

\section{Task Experiences on LHRDs}

Bezerianos and Isenberg [8] studied the perception of angle, length, and area when close to, and far away from, the LHRD, finding that people are more accurately able to compare these variables when further away from the screen than when close or even when moving to and fro. Additionally, lower placed screens seem to feature more perceived distortion than screens at chest height and above. Yost et al. [29] note that LHRDs with pixels in excess of visual acuity require physical navigation to fully explore displayed information. Their study found that even with the extra time required for the physical navigation task, performance was more efficient and in some cases more accurate.

Czerwinski et al. [13] found that the wide field-of-view provided by large displays improves task performance of both men and women and, additionally, narrows the gender gap in performance on the spatial task of navigating a virtual world.

A concern is that the move to LHRDs will enact changes in perception in comparison to visualizations presented in smaller areas on desktop displays. Endert et al. [15] examined how the use of different visual variables (colour, length, slope, and position) causes differences in physical navigation and task performance in front of large displays. They found colour to be a preferred encoding, likely due to its positionindependence as well as the ease with which the visual system aggregates colour as one moves further away.

\section{Utilizing LHRD Space}

Studies [5, 9] have compared everyday usage of LHRDs to that of standard desktop scenarios for usual applications such as word processing, reading papers, and data analysis. LHRDs were preferred by participants in both sets of studies, noting their strengths in task switching, peripheral awareness, collaborative work [5], rich-information tasks, and enhancement of focus due to the ability to physically separate focal (working) and peripheral (supporting) information [9].

Knudsen et al. [20] performed an exploratory study of large display interaction through groups of two or three using whiteboards. They noted persistent use of space, data comparisons performed both side-by-side (in space) and sequentially through interaction, temporary use of large spaces to select/modify data, use of space to leave a history of analysis, and physical movement to achieve overview (and vice versa to achieve focus).

Andrews et al. [2] completed two observational studies; one comparing the use of space on a standard desktop display to that of an LHRD and the other observing professional analysts' usage of space with an LHRD. From these observations the authors noted the participants' use of space as external memory where full documents were accessed by location and physical navigation of the screen, rather than virtual navigation of the computer's file system. Participants also made use of operations such as ordering, proximity, stacking, and alignment as a flexible semantic layer of information. Andrews and North built upon these findings to develop an application [4] that uses the space in LHRDs to support sensemaking analysis.

\section{Collaborative Work on LHRDs}

By increasing resolution and size, LHRDs provide room to lay out, organize, and structure applications or information in general for both individuals and collaborating groups. Guimbretière et al. [16] note that LHRDs are suited for collaboration, bringing a digital interface to the utility of a whiteboard.

Bradel et al. [10] studied pairs of participants engaged in visualization or text analysis tasks. With independent mice and keyboards, participants had the opportunity to work independently or collaboratively. Pairs that collaborated closely shared the display space and produced higher quality results, whereas pairs that worked independently separated the workspace into individual and shared territories. Jakobsen and Hornbæk [19] studied a pair task on a multitouch LHRD. With the close proximity to the screen, participants were able to switch fluidly between parallel and joint work but seemed to share the display without territoriality.

Andrews et al. [3] also raised the question of whether LHRDs with more than 4.35 million pixels are wasting resources. They noted that physical navigation to concentrate on important areas, as well as group collaboration, both provide scenarios where even higher pixel counts remain useful.

Beaudouin-Lafon et al. [7] wrote about the use of their LHRD by experts in a variety of science fields including astrophysics, particle physics, chemistry, molecular biology, neuroscience, mechanical engineering, and applied math. In providing an overview, the authors noted the following common applications of their display wall: navigating a single, very large object (such as a complicated 3D molecule or an extremely large image), comparison of a large number of related visuals (such as photographs or information visualizations), and juxtaposition of data from different sources (e.g., mixes of applications, articles, data tables, notes, and so forth). For our purpose, we conducted a study to investigate how people from various disciplines - including humanities, design, and social sciences - found the LHRD useful in their work. To create a natural and close to real life environment, we allowed our participants to perform self-directed tasks with their own data.

\section{Summary}

Investigators [6, 12, 21, 25] have studied how LHRDs are useful in many respects, such as changes in performance of perception, collaboration, and analysis tasks. However, most of these studies follow a predefined procedure, asking participants to perform series of directed tasks, answering the specified questions about interaction performance [23, 6] and the tasks and applications studied [2, 19, 24, 29]. In contrast, our work focuses on naturally arising usage and application of LHRDs in an open, academic setting. In this work, we asked participants to bring their own data, show us their use of LHRDs in a self-directed procedure, and describe their previous experiences using the LHRD. 


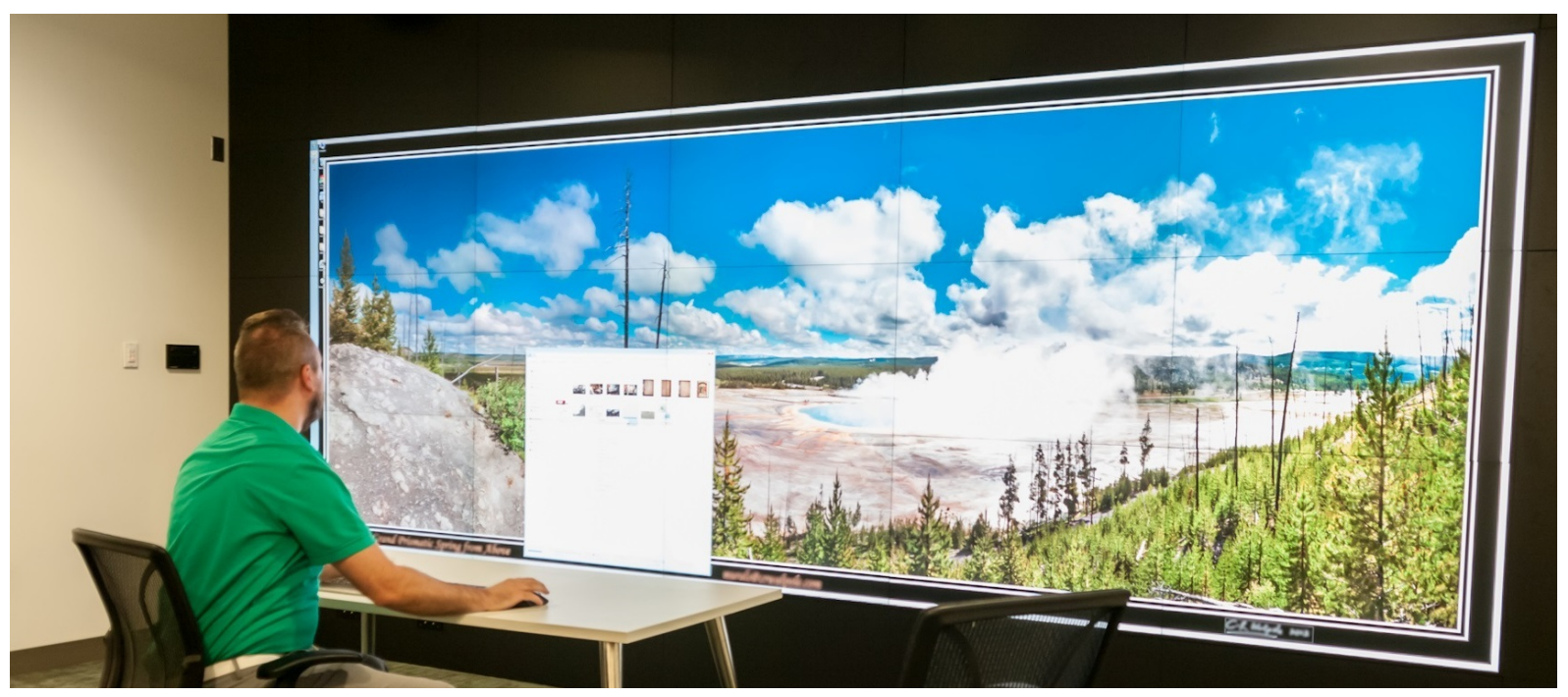

Figure 1. The 34.5 million pixel display wall used in the study.

\section{STUDY DESIGN}

To gain a rich picture of real-world usage and potential of LHRDs, we conducted semi-structured interviews with researchers in context of a mega-pixel display while displaying the researchers own large or high-resolution data. We analyzed these interviews using qualitative methods. Our study goal was to better understand whether there are advantages in the sheer scale of displays: do size (physical dimensions) and resolution (pixel count) hold advantages for people in a variety of disciplines? Since much of the driving force for the creation of these displays has come from the natural sciences, we were particularly interested in investigating whether any possible advantages extend into other disciplines.

\section{Participants}

We recruited the 14 participants, who work with high resolution data (large datasets or images bigger than 2 mega-pixels), through word-of-mouth and email across the university. Participants were asked to bring large datasets or images that they had explored previously on their typical office displays.

From these 14 participants, there were five females and nine males whose ages ranged between 26 to $66+$ years. All participants were university researchers who regularly worked with large digital data. More than half of the participants were professors. We obtained a good interdisciplinary mix with people from a variety of research areas including: Medieval \& Renaissance Literature and Art, Multi-resolution Data Vis, Environmental Psychology, Digital Humanities, Ecology Maps GIS, Landscape Visualization, Bio-inspired Design, Medieval Manuscripts, Photography Design, Sociology, Radiology/Psychology, Virtual Reality \& 3D Imaging Architecture Planning, Computer Graphics, and Environmental Design.

The LHRD at our academic institution is open to all faculty and graduate students. Some participants had already used the LHRD in their research and were familiar with the general set up and with how their data appeared on the screen, while others were using it for the first time.

\section{Set up}

The LHRD is $4.95 \mathrm{~m} X 1.85 \mathrm{~m}$ and made with 15 rear projection screens. Each projector has a 1920 X 1200 resolution for a total of 34.56 million pixels ( 9600 X 3600 pixels at $50 \mathrm{DPI})$. The wall is operated by a single PC with dual Xeon E5505 processors, 96 GB RAM, and four NVIDIA Quadro K5000 GPUs running a Windows 7 operating system. The room was configured as shown in Figure 1, with a wireless keyboard and mouse on a desk. The room offered a quiet working space similar to an office or research space where researchers would ordinarily explore their data. The front of the desk was positioned roughly one meter away from the display. Participants were free to get up and walk around as needed. A variety of applications (e.g., Photoshop, GIMP, Mathematica, Excel, InDesign, Illustrator, ArcGIS, MeshLab, Unity) were installed on the system to allow participants to easily access files with familiar software. This familiar interface and software environment on the LHRD provides an extremely easy transition from standard desktop environments.

\section{Procedure}

After showing participants that the LHRD operates like a Windows desktop, we asked participants to put their own data on the screen and to work with and explore it at their own pace (for up to half an hour). Then we started the semistructured interview portion, asking questions prepared in advance as well as questions based on the participant's data, actions, and responses. Initial questions were structured around three main categories: participants data and how it was used, envisioning tasks on LHRDs, and specific activities participants would undertake when working with their data. Followup inquiries explored how participants conducted research, processes they used when working with their data, and any insights and discoveries they had made. We also discussed the participants' experience of looking at their own data on 
the LHRD, possible tasks they can perform on the display, and any future possibilities with the display. Intentionally, in our study, we did not start by asking our participants to perform any particular tasks; instead, we asked them to describe different "actual" usage of the LHRD for their work and the tasks for which they found the LHRD useful.

\section{Data Collection and Analysis}

Two experimenters were present in the interviews at all times and took field notes. We video and audio recorded the interviews. The data was transcribed and then analyzed as follows. First, we identified the main categories together based on the interviews. Second, we used open coding and coded the video transcripts line by line. One researcher independently coded the transcripts and two other researchers coded a subset of the transcripts. After this phase, we cross checked our codes to reach an agreement. From this step, we gathered approximately 500 codes. Working with this extensive list of codes, we used iterative affinity diagramming and group discussions to identify a total of twelve different participant activities. We grouped these activities into four activity classes: immersive observation, observation strategies, collaboration, and education (see Table 2).

\section{RESULTS}

Our interviews have shed light on the usage of LHRDs by people in disciplines generally not targeted by LHRD designers and creators: disciplines including the humanities, social sciences, art, and environmental design. None of our participants thought of their data as "big data", which has been imagined to be the most frequent use case for LHRDs [27]. Our participants' data was usually highly detailed image data (10 of 14 participants). We also had a wide range of usage experience with the LHRD ranging from people who had made publishable discoveries; to people for whom the LHRD had become part of their research processes; to people who had little experience with the LHRD, and including people who were bringing their data to the LHRD for the first time. We have speculative comments from all participants, provided incontext of the display while examining their own data, about ways they envisioned using the LHRD. In addition, we have a strong set of experience-based comments from the participants who had used the display in their own work, providing a reliable picture of the benefits they had already experienced from using the display.

We first note the range of disciplines and range of types of data studied because we think that usage across disciplines is an important part of our results. We then show the results of our coding, and list the data insights and discoveries that participants told us about.

\section{Multiple Disciplines and Data Types}

Table 1 shows the wide disciplinary spread amongst our participants. Most of our participants work in disciplines outside of the natural sciences. All of our participants had previously used their data on typical office displays (with less than 5 million pixels) or using projectors that had, at best, HD resolution.

\begin{tabular}{|l|l|}
\hline Discipline & Data discussed \\
\hline \hline GIS Librarian & Historical maps \\
\hline Literature & Old manuscripts \\
\hline Urban Design & 3D city neighborhood models \\
\hline Computer Graphics & Satellite city photo \\
\hline $\begin{array}{l}\text { Medieval and Renaissance } \\
\text { Literature and Art }\end{array}$ & Paintings \& engravings \\
\hline Urban Design & 3D city models and layered city maps \\
\hline Art & Panoramic photos \\
\hline Computer Graphics & Large photographs \& satellite imagery \\
\hline Environmental Design & 3D models \\
\hline Art, Design, \& Education & Online magazine \\
\hline Environmental Design & 3D modeling \\
\hline Radiology/Psychology & 2D \& 3D brain images \\
\hline Sociology & $\begin{array}{l}\text { Network graphs \& spreadsheet data } \\
\text { analysis }\end{array}$ \\
\hline Literature & Old manuscripts \\
\hline
\end{tabular}

Table 1. Participants' research area and the data type discussed.

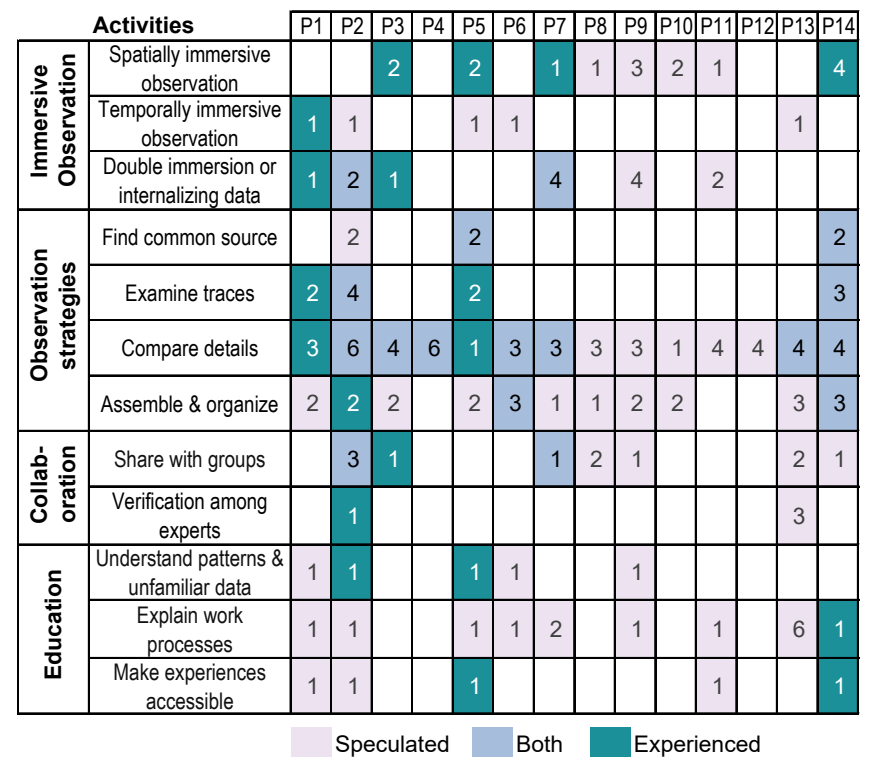

Table 2. Coding results: Numbers shown are the count of times each participant mentioned the activity. Colours indicate whether mentions discuss activities experienced, speculated upon, or both situations.

\section{Activities on the LHRD}

During our interviews, we asked our participants to describe the range and types of tasks they used on the LHRD, noting which they found useful in their work. Since we are interested in both how the LHRD is currently being used and whether new or different interactions might be of interest, we coded both for the interaction they had experience using and those that they speculated about: the ones they were interested in being able to use. Table 2 shows these results. The left column holds the resulting categories for which we coded.

\section{LHRD Aided Discoveries and Insights}

Seven of our fourteen participants had either previously, as part of their own research, or during the study made discoveries with the LHRD. For several of these people the discoveries were publishable. They attributed these discoveries to their use of the LHRD because they had previously explored 

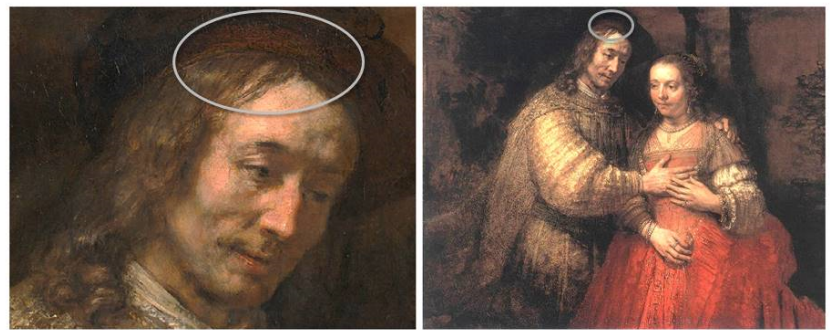

Figure 2. P5 discovered a yarmulke under a hat in The Jewish Bride [26].
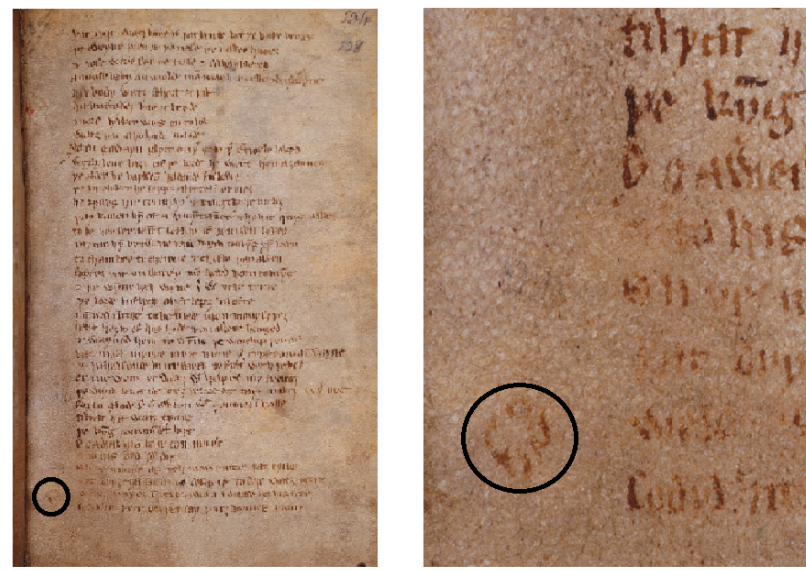

Figure 3. P14 discovered an asterisk in the form of a rose petal on a page of a manuscript. () The British Library Board, Cotton Nero A.x. (art. 3) f. 104/108.

this same data on standard-sized screens in their regular office environments without this success.

The discoveries mentioned to us include:

- Medieval literature and art: A yarmulke hidden under a hat was noticed in one painting, and traces of a mustache on a model across several paintings. This credibly added to the body of evidence that the same model had been used for multiple paintings; the yarmulke (Figure 2) also gave further clues as to the model's identity.

- Medieval literature: On examining old manuscripts, several annotations made on a manuscript were discovered: a manicule, a half-finished manicule that s/he had originally thought was an insignificant scribble, and an asterisk in the form of a rose petal (Figure 3).

- Medieval literature: Another related to the variations in the "aspect" of a manuscript's pages (the particular characteristics of the handwriting across a page), which were qualified as not enough for a separate publication, but said "it will definitely form a paragraph in our introduction to the online facsimile and therefore constitutes knowledge."

Besides these publishable discoveries, many participants noticed new aspects of their data, which were intriguing and sometimes useful for their research. These included:

- Digital humanities: A manicule (an annotation in the shape of a hand) was noticed on a manuscript page when s/he was using the LHRD to translate the page from middle to modern English.

- Urban design: Various imperfections in a 3D neighbourhood model s/he had created were noticed and subsequently corrected.

- Computer graphics: New details were noticed in satellite imagery that s/he was using as a reference for 3D models, such as the start point of a sharp slope, a depot in the back yard of a building, and building textures.

- Art: Imperfections in his/her panoramic photographs were noticed.

- Environmental design: The participant felt that s/he developed a better sense of how shade affects pedestrians in a $3 \mathrm{D}$ model of a neighbourhood.

\section{DISCUSSION}

Our qualitative, interview-based approach uncovers a multifaceted picture of several researchers' actual usage of an LHRD, which, in addition to the purely technical aspects of the display itself, includes aspects of time, environment, and data. We discuss how participants viewed the LHRD's role in supporting multiple aspects of their work: its immersive environment, its support of particular observation strategies, its support of collaboration, and its unique benefits for education.

\section{Immersive Observations}

One of the more striking results of our interviews was that two participants had made three publishable discoveries while using the LHRD. These discoveries all involved seeing things that no one had seen before in high-resolution photographs of old manuscripts or paintings. There were also many other explicitly mentioned personal discoveries and discoveries that might yet be verified as significant. To better understand the role of the LHRD in these discoveries, we describe how our participants talked to us about observation. Observation was a key concept addressed in every interview. This is not surprising because observation has long been known to be a deeply important process to discovery. Since Aristotle, and perhaps before, scientific discovery has been based on observation. Also, as we have seen in this study, observation is also important to discovery in other disciplines. In their comments there are two levels at which observation is mentioned. In terms of the LHRD, all-encompassing observations, or immersiveobservations, are composed of spatial and temporal factors. More specific, detailed observations result from smaller more focused observation strategies.

\section{Spatially Immersive Observations}

One potential factor was the immersive experience of being in the room with the LHRD. For instance, P5 cited the darkness and isolation of the room as allowing for a different feeling than even being in a museum with a painting: "in this room we can be very intimate with the great work of art." He also spoke to the quality of the image: ".... whereas this room can be very dark and we can appreciate pixels." $\mathrm{P} 14$ found the 
flexibility of spatial arrangement allowed him to better understand differences in aspect, which refers to the overall characteristics of handwriting on a line or page. Aspect can differ from scribe to scribe and even based on the tools the scribe is using. Previously, the best way to see differences in aspect was to flip through the pages of the physical manuscript; the LHRD allowed him a more beneficial view of the data: "Because in the British Library, they are strangely resistant to you pulling the pages out of the manuscript and putting them side by side [laughs]. So we can only do that with imaging. And only when you can look at the whole page can you really get some of these things about aspect, so that's another thing that we've been using this [LHRD] for."

\section{Temporally Immersive Observations}

Another factor may have been that researchers can use the room for a long stretches of time. Although we do not have specific data about how long it took to make these discoveries on the LHRD, we do have some indication of the amount of time tasks such as these take. P5 cited one observation task that involved him spending eight hours looking at one object depicted in a painting before finally seeing relevant details. This is consistent with $\mathrm{Bi}$ and Balakrishnan's [9] report that LHRD immersion assisted their study participants in focusing on attentive tasks. This recalls an anecdote about the work of the artist David Hockney, who along with physicist Charles Falco proposed the theory that the sudden transition in realism in paintings in the 1400s was due to the use of optical aids such as the camera obscura [18]. Hockney used this technique of long-term observation as part of his discovery process. He arranged print-outs of 400 years of paintings in chronological order on a large wall in his studio, which he called his "Great Wall". In the documentary [28] about his work, he notes, "the wall was necessary because I could then sit back and scan centuries of Western painting". In some ways the LHRD is similar to Hockney's Great Wall, an environment conducive to long periods of detailed observation.

\section{Double Immersion or Internalizing Data}

Participants talked about the combined effect of spatial and temporal immersion as internalizing the data. Seeing digital artifacts on the LHRDs was a different experience from seeing them on standard displays. Participants variously described it as "feeling like you are there in the data"(P11), "you can get right up to it"(P9), and being "immersed"(P07). This experience allows participants to internalize patterns and forms inherent to their digital artifacts. For example, while learning to translate medieval manuscripts, $\mathrm{P} 2$ displayed them in order to familiarize herself with the way language looked on the page. She said: "I am not familiar with old English the way that specialists might be, so for someone like me, it was very helpful to have this resource. Because I was so unsure and I had to basically learn the language and what it looks like. And that was impossible for me on my own laptop ... the letters were [so small] you know, and I just couldn't do it. For this, I actually got to the point where to begin with, it was painstaking and took me forever, where in here [the LHRD] I was able to do it a lot quicker and to learn a lot more about the letters, I think, seeing them in a larger format. So it was great that way."

\section{Observation Strategies}

In addition to the high level immersion experienced by participants, they also utilized low level observation strategies to examine and explore their data.

\section{Find Common Source}

One task common among the historians we interviewed was to search for commonalities across distinct artifacts. For instance, P5 was searching for evidence that a collection of paintings depicted the same model. He displayed photographs of two paintings and two etchings, magnified the man's face in each one, then pointed out details common to both, such as a faintly visible scar on the man's face, mustache, and shape of nose and chin. These details, particularly the scar and mustache, were difficult to spot at lower magnifications. Similarly, P14 closely examined the details of the lettering across many pages of a manuscript, searching for evidence that the entire text had been written by the same scribe. "You can take pieces of each of those [pages] and have a word for each that it is the same and see, ok, this is clearly the same writing so it is the same scribe, which is a very interesting finding in medieval literature."

\section{Examine Traces}

Old artifacts frequently have faded, lost ink, or brightness due to the effects of time, dust, and human contact. However, reading and understanding the original traces is valuable for further investigation. Four participants in our study found the LHRD useful for deciphering these traces in their own work. P1 was able to closely follow traces of pencil sketches on an old city map to determine previous planning on the map that could help her in the process of gathering data for urban designers. P2 and P5 adjusted contrast, brightness, and hue (activities that they had previously used on standard displays) to reveal faded, difficult to see details such as identifying Middle English letters (P2) and specific facial characteristics (P5).

In addition, P14 was investigating old manuscripts to find how and by whom they have been annotated. He put up the manuscript on the screen to magnify the details and examine several faded manicules in the manuscripts; meanwhile he found several unexpected manicules he had not seen before. It should be noted that size and high resolution of the screen was the main reason for this finding.

\section{Compare Details}

Having access to LHRDs allowed participants to compare multiple items with viewable detail. Although this simple task was common to many participants, there are variations in the way it was actually performed: laying out multiple pages of manuscripts at once, comparing different versions of translations, and zooming in on paintings and searching for details.

The high resolution and large size of the LHRD supported these detailed tasks that involved spotting small differences. For instance, participant P2, was interested in distinguishing "minims", which are similar-looking blackletter-style letters such as $i, m$, or $n$, in a word or sentence. This task was only possible when s/he could see details of the letters set in the detailed context of the word or sentence. She achieved this by magnifying the whole page and changing colour tone to 
increase the contrast. This task was useful in for translation purposes. De la Flor et al. [14] have studied this type of task of transcribing ancient texts in detail, supporting this task with an overview+plus detail system presented on a standard resolution projector screen. However, they noted issues with the quality of the projection image as well as lack of space to compare images and juxtapose text and notes. Spotting a small difference between artifacts is also a common procedure with scientific data, especially for finding relationships or patterns. For example, the psychology participant, who compared multiple MRI brain images to find relationships between regions of the brain network, says she "needs to see the context of the brain to see whole region it is in" which was possible with the large high resolution display.

There also is a reliance on "foot-powered" zooming [2, 20, 21] where details are examined through physical rather than virtual navigation. P13 noted the utility of this zooming mechanism in a collaborative setting "If I was illustrating to someone I could say look at [the network graph's node] number 19 and I could walk over there .. here is number 19. It's supposed to have three connections, does it? Let's check them."

\section{Assemble and Organize}

Assembling heterogeneous data sources, such as the juxtaposed text and notes mentioned by de la Flor et al. [14], was another common activity noted by other LHRD researchers $[2,7]$. Participants mentioned several examples of using the space of the wall to layout and interpret data, creating a single, contiguous presentation from many sources. As stated by P3, "We are also working on dash boarding and trending information and trying to pull all different components together from four different databases into a single reporting structure. Being able to see the different components and relate the data, I think this would be pretty useful for that."

\section{Collaboration}

Another theme of participants' comments was in making use of the LHRD space and resolution to collaborate with groups for different purposes, including collaboratively solving problems, collaboratively comparing activities during translation and referring to additional materials while translating, and collaboratively viewing data with colleagues for analysis and verification.

\section{Share With Group}

Many participants used the LHRD to share artifacts with others in scenarios that require space and high resolution to be able to see details. P14 stated this directly, "It would be nice if we could bring everyone in here to show them how does it look like on this big screen because sometimes in the points of comparison are not so obvious when you are looking at that little square." P9 noted that ten years ago for big projects he printed everything out, covering an entire wall. Now, with slide presentations, people lose track once the slides pass, whereas on the LHRD you can display all this information at once.
Another participant, P2, who had come with her colleagues previously, mentioned how their collaboration process changed once they started to use the LHRD. They had been trying to solve problems on their laptops. However, they suddenly realized they could use the LHRD to share the problems' data with everyone and collaboratively solve the problem. This collaborative use may point towards the future potential of LHRD usage once these displays have been more widely spread and the potential usages have been better identified and supported.

\section{Verification Among Experts}

Verification among colleagues is also noted as important and this process needs multiple people, sometimes with different backgrounds gathering and comparing various items of data. The LHRD was helpful in these situations for displaying material in a manner that lets everyone to refer to it, P13: "And also what we really want to do with that is have multiple people on it so to be able to have three people in here and literally to go through every single record and confirm, all three of us confirm, I think that is the same author."

\section{Education}

Education is rarely mentioned in the HCI literature on LHRDs, but our participants in general were enthusiastic about the utility of the display for educational purposes, volunteering many ideas about how they have used or would like to use it for education. While this is not entirely unexpected considering that most of our participants were educators or students, their enthusiasm revealed a desire to share digital artifacts in higher fidelity and at a larger scale than they are currently able in conventional classrooms. Some had already used the screen for educational purposes, both for learning by themselves, for teaching students to analyze paintings, for student presentations, and for sharing research processes and results with graduate students. Others described to us how they intended to use the screen for education or what they thought it would be useful for.

\section{Understand Patterns and Unfamiliar Data}

Several participants mentioned that it was their experience that students have a hard time understanding patterns in unfamiliar data, and that being able to show them the entire view of the data while explaining smaller parts was very useful. This was common across design-, process-, and observationoriented education. One design-oriented researcher, P9, told us that he would like to be able to show students full-scale maps of various city infrastructures: "I have been working with these sorts of images for 17 years, so for me I can do the zooming in and out on my computer. I have done it so many times that I'm used to it. I paint a mental picture of the whole city but even then it is still hard if you are unfamiliar with the place. But for students it is really hard. So to be able to see the whole screen and show them patterns, that could be a really good teaching tool to be able to do that on the screen." The usefulness of this sort of situation was confirmed by P2, who you may recall used the LHRD as a student to "learn how [Old English] looks" - in other words, to internalize the forms of the "data" - the language - that s/he needed to work with. 


\section{Explain Work Processes}

There were several comments about the screen being useful for explaining processes to students. P14 used it to explain to a team of graduate students how he had found the manicules in the manuscripts. P13 wanted to bring new researchers in her lab to the room to explain the analysis process they would be using for the data; this would allow all of them to learn the process consistently and simultaneously. P13: "When we did that project with grad students that was so useful to be able to all look at the data together, it is so much better ... to be able to say does it match does it right over here? We were combining variables, all that stuff, it was really useful." P7, with the photography background, stated that to find the perfect combination of lenses and sizes, it is important for his students to be able to see the process and compare the results of different combinations, which was possible on the LHRD due to the amount of space.

The resolution of the LHRD allows many people to simultaneously see the details of what is happening on-screen. This can be helpful in the classroom, as noted by P11, who compared the LHRD to the projectors typically used in classrooms: "Yes, I think if you are doing any sort of demoing, especially with GIS, one of the big failures of ordinary projection is that they can't read anything. Even if you zoom in, they can't read it, it is just blurry."

\section{Make Experiences Accessible}

The screen could also be useful for allowing students to have experiences they otherwise could not have. For instance, it can provide access to paintings or items from a museum in a different city, which students who did not have access to as noted by P5: "...the impact here is overwhelming because it captures the structure... [and] the size of the painting on the wall is important, here it is quite good and students can actually relive it."

\section{LESSONS LEARNED}

In this section, we present the most important lessons we learned about the naturally arising use of LHRDs across multiple disciplines. This includes pointers to as of yet under explored avenues for further research related to LHRDs and the types of interaction that might lead to richer support of research processes in multiple disciplines.

\section{Size Plus Resolution}

The size and resolution of the display helped the participants identify details and/or flaws in their data or in general gain more insight about their data. Many of our participants said that there were other options, projectors and LCD panels, for simply viewing their data on large format displays, but that simply the larger format was not sufficient. They needed the size plus resolution. It is important to emphasize that all the data belonged to and had previously been worked with by the participants on standard displays. Consequently the LHRD was an instrumental element of the new discoveries.

Participants also mentioned that it is natural to take advantage of the size of the display to collaborate in a group. For instance, P2 stated: "I think people don't necessarily know and even when they hear about it, it is like OK you can make the images bigger, it is great, and you don't really think about it in a way of collaborative until you are in here."

\section{Use of LHRDs in multiple disciplines}

Our initial questions centred on whether LHRDs were of interest or in use in disciplines outside of the natural sciences. The answer is a resounding yes. Every single one of our participants discussed multiple usage scenarios and saw existing benefits and potential for new benefits to accessing their data on the LHRD. They were able to use the LHRD as a tool to aid in discovery in their research and design processes. Moments of insight occurred multiple times during the interviews suggesting that this technology may be game-changing for work across many disciplines that involve large scale information.

\section{The Process of Observation}

A particularly fascinating aspect of this study is that our participants spoke in detail about how the use of the LHRD helped their process of observation. There were two important parts to their observation process: factors that helped to immerse them in their data; and the more specific lower-level observation strategies that they used during observation. The immersive factors were explicitly connected to:

- Size: Factors included seeing data at normal scale, and magnified.

- Local and global detail: Every single participant talked about the importance of being able to see the details; both globally in terms of details in an entire image and locally when zooming to specific areas.

- Time: This interesting factor is not a factor of the display but of the policy around its use: it was relatively simple for researchers to arrange for prolonged access.

In combination these factors produced what some of the participants referred to as a data-immersive experience. The lower level observation strategies included:

- Finding a common source: Several participants find evidence of a common origin some data aspects that might be repeated, perhaps with variations.

- Examining traces: This involved looking for particular details but also often about looking for details of previous human interaction with the data.

- Assembling and organizing: The ability to arrange and rearrange their data played an important role.

- Compare details: The observation strategy was discussed by all participants and included many variations.

Through using these lower-level strategies our participants made many discoveries in their data, some of which have already led to publications. The LHRD provided people with a fresh, immersive experience that helped them to identify details or flaws in their digital artifacts.

\section{Future directions for LHRD Interaction Research}

The researchers we spoke to had used and/or planned to continue to use the LHRD as a tool for discovery. Even those 
using the display for the first time noticed various details in their own digital artifacts that they had not seen before. The impact of the LHRD on expanding knowledge could be comparable to that of magnifying lenses, which, when introduced, allowed people to see their objects of study in a completely different way than previously.

It is particularly interesting that the researchers we spoke to had used - and planned to continue using - the LHRD as a tool for discovery despite the fact that its interaction was essentially that which is available on desktop-sized displays. This limitation of the LHRD to mouse and keyboard was not ideal, as often participants wanted to touch the board to point something out, but the physical transition between the table with the keyboard and the screen was cumbersome. Using regular software such as Adobe Illustrator, which was not designed for such a large screen, was difficult due to exceedingly small interface elements. We acknowledge that there is richer interaction design available than we had access to for our LHRD, such as touch-based interaction [2, 3, 4], gesturebased interaction and multi-device interaction [7].

There are also positives to this set up. It allows people with limited technical backgrounds to easily use familiar desktop software and related interaction mechanisms. The keyboard and mouse on the table as shown in Figure 1 encouraged participants to look at the overview of the screen. This likely led to some of the observed interaction patterns noted previously, such as the foot-powered zooming. Importantly, these limitations did not prevent our participants from finding uses for the display and making discoveries.

Based on these observations, we would like to encourage more interaction design for LHRDs, specifically for the observational practices described by our participants. There is a strong need for better observational tools that support the noted observation strategies. In particular the comparing details activity included many more scenarios than the commonly discussed need for detail-in-context. In comparison to a large-sized display with low resolution or a high resolution, small-sized display, the LHRD provides readily visible access to both detail and context simultaneously. For instance, participants talked about:

- Having access to local details inside a detailed context.

- Comparing full details of two or more distinct data images.

- Comparing small parts at full zoom.

Lastly, we do not want to imply that the activities we observed were the only uses of the LHRD, merely that these are additional, underexplored, but real uses.

\section{LHRDs' potential to create rich educational experiences}

While some of the researchers we talked to felt they could cope with some of their large data on their own laptops, as they had the experience to develop useful mental models of their data, they were very keen to use the LHRD to aid in sharing their knowledge with others. This potential of the LHRD for educational purposes also opens up new questions for future research. That size and resolution together make it possible to share richer and more detailed digital artifacts with students, enriching the educational experience.

\section{Systems conducive to observation for discovery}

We would like to encourage the design and building of systems conducive to observation. We recognize that these are difficult to study because they require long-term use. However, it was clear from our interviews that observation is extremely important to making discoveries across a variety of disciplines, and that this is worth supporting. For our participants, the new perspective provided by the combination of size, resolution, time, and basic tools (image manipulation, zooming, and spatial arrangement) helped make their observation-based discoveries possible. There are bound to be additional, less apparent observation processes at work that could be better supported by LHRD systems.

\section{CONCLUSION}

We have presented an exploratory study of the use of large, high resolution displays (LHRDs) across multiple disciplines. We discovered that these LHRDs are successfully being used for research across many disciplines outside the natural sciences. Perhaps most importantly, from our participants we learned that - while they had access to large or highresolution displays in their labs and offices - it was the combination of a very large display with high resolution that made it worth going out of their way to use the LHRD.

We also learned interesting details about observation strategies, which we hope will motivate new interaction research. These strategies include: looking for common factors, examining traces, flexible assembly and organization, and the importance of comparing details. In particular, our participants valued local details set in global details, comparisons done at full scale, and comparisons done at high zoom. The wide range of people taking advantage of the capabilities of the LHRDs suggests that designing for these displays needs to go beyond considering how scientists and data analysts would use them to include the particular needs of other diverse disciplines.

Our participants gained various types of insights from using the display, including publishable discoveries. They shared their experiences regarding immersion, observation strategies, collaboration, and education. We hope that the clear significance of LHRDs in promoting observation and discovery will encourage more interaction research in this area. We reiterate that it was neither the size nor the resolution on their own that promoted the types of insights and experiences we saw in the use of the LHRD; it was size plus resolution that made the difference.

\section{ACKNOWLEDGMENTS}

This research was supported in part by AITF, NSERC, GRAND, SurfNet, and SMART Technologies. We would like to thank our participants and reviewers for the expert knowledge they brought to this project. 


\section{REFERENCES}

1. Alphonse, G. A., and Lubin, J. Psychophysical requirements for tiled large-screen displays. In Proc. SPIE, vol. 1664 (1992), 230-240.

2. Andrews, C., Endert, A., and North, C. Space to think: Large high-resolution displays for sensemaking. In Proc. CHI, ACM (2010), 55-64.

3. Andrews, C., Endert, A., Yost, B., and North, C. Information visualization on large, high-resolution displays: Issues, challenges, and opportunities. Information Visualization 10, 4 (2011), 341-355.

4. Andrews, C., and North, C. Analyst's workspace: An embodied sensemaking environment for large, high-resolution displays. In Proc. IEEE VAST (2012), 123-131.

5. Ball, R., and North, C. Analysis of user behavior on high-resolution tiled displays. In Proc. INTERACT, Springer (2005), 350-363.

6. Ball, R., North, C., and Bowman, D. A. Move to improve: Promoting physical navigation to increase user performance with large displays. In Proc. CHI, ACM (2007), 191-200.

7. Beaudouin-Lafon, M., Huot, S., Nancel, M., Mackay, W., Pietriga, E., Primet, R., Wagner, J., Chapuis, O., Pillias, C., Eagan, J., Gjerlufsen, T., and Klokmose, C. Multisurface interaction in the wild room. Computer 45, 4 (2012), 48-56.

8. Bezerianos, A., and Isenberg, P. Perception of visual variables on tiled wall-sized displays for information visualization applications. IEEE Trans Vis Comput Graph 18, 12 (2012), 2516-2525.

9. Bi, X., and Balakrishnan, R. Comparing usage of a large high-resolution display to single or dual desktop displays for daily work. In Proc. CHI, ACM (2009), 1005-1014.

10. Bradel, L., Endert, A., Koch, K., Andrews, C., and North, C. Large high resolution displays for co-located collaborative sensemaking: Display usage and territoriality. Int J Hum-Comput St 71, 11 (2013), 1078 1088.

11. Cruz-Neira, C., Sandin, D. J., and DeFanti, T. A. Surround-screen projection-based virtual reality: The design and implementation of the cave. In Proc. SIGGRAPH, ACM (1993), 135-142.

12. Czerwinski, M., Smith, G., Regan, T., Meyers, B., Robertson, G., and Starkweather, G. Toward characterizing the productivity benefits of very large displays. In Proc. INTERACT, IOS Press (2003).

13. Czerwinski, M., Tan, D. S., and Robertson, G. G. Women take a wider view. In Proc. CHI, ACM (2002), 195-202.

14. de la Flor, G., Luff, P., Jirotka, M., Pybus, J., Kirkham, R., and Carusi, A. The case of the disappearing ox: Seeing through digital images to an analysis of ancient texts. In Proc. CHI, ACM (2010), 473-482.
15. Endert, A., Andrews, C., Lee, Y. H., and North, C. Visual encodings that support physical navigation on large displays. In Proc. GI, CHCCS (2011), 103-110.

16. Guimbretière, F., Stone, M., and Winograd, T. Fluid interaction with high-resolution wall-size displays. In Proc. UIST, ACM (2001), 21-30.

17. Hereld, M., Judson, I., and Stevens, R. Introduction to building projection-based tiled display systems. IEEE Computer Graphics and Applications 20, 4 (July 2000), 22-28.

18. Hockney, D. Secret Knowledge (New and Expanded Edition): Rediscovering the Lost Techniques of the Old Masters. Viking Studio, 2006.

19. Jakobsen, M. R., and Hornbæk, K. Up close and personal: Collaborative work on a high-resolution multitouch wall display. ACM Trans. Comput.-Hum. Interact. 21, 2 (2014), 11:1-11:34.

20. Knudsen, S., Jakobsen, M. R., and Hornbæk, K. An exploratory study of how abundant display space may support data analysis. In Proc. NordiCHI, ACM (2012), 558-567.

21. Liu, C., Chapuis, O., Beaudouin-Lafon, M., Lecolinet, E., and Mackay, W. E. Effects of display size and navigation type on a classification task. In Proc. $\mathrm{CHI}$, ACM (2014), 4147-4156.

22. Max, N. L. Computer graphics distortion for IMAX and OMNIMAX projections. In Proc. Nicograph (1983), 137-159.

23. Robertson, G., Czerwinski, M., Baudisch, P., Meyers, B., Robbins, D., Smith, G., and Tan, D. The large-display user experience. IEEE Computer Graphics and Applications 25, 4 (July 2005), 44-51.

24. Shupp, L., Andrews, C., Dickey-Kurdziolek, M., Yost, B., and North, C. Shaping the display of the future: The effects of display size and curvature on user performance and insights. Human-Comp Interaction 24, 1-2 (2009), 230-272.

25. Tan, D. S., Gergle, D., Scupelli, P., and Pausch, R. Physically large displays improve performance on spatial tasks. ACM Trans. Comput.-Hum. Interact. 13, 1 (Mar. 2006), 71-99.

26. van Rijn, R. H. Isaac and Rebecca, known as 'The Jewish Bride'. Painting, oil on canvas, c. 1665 - c. 1669.

27. Vogt, K., Bradel, L., Andrews, C., North, C., Endert, A., and Hutchings, D. Co-located collaborative sensemaking on a large high-resolution display with multiple input devices. In Proc. INTERACT, Springer (2011), 589-604.

28. Wright, R. David Hockney's Secret Knowledge. British Broadcasting Corporation [TV Movie], 2003.

29. Yost, B., and North, C. The perceptual scalability of visualization. IEEE Trans Vis Comput Graph 12, 5 (2006), 837-844. 